\title{
FACTORES DE RIESGO MATERNOS ASOCIADOS A LA PRESENTACIÓN DE RECIÉN NACIDOS MACROSÓMICOS EN EL CENTRO MÉDICO NAVAL CIRUJANO MAYOR SANTIAGO TÁVARA EN EL PERÍODO JULIO 2014 A JULIO 2016
}

\author{
MATERNAL RISK FACTORS ASSOCIATED WITH THE PRESENTATION OF MACROSOMIC NEWBORNS AT THE NAVAL \\ MEDICAL CENTER CIRUJANO MAYOR SANTIAGO TÁVARA IN THE PERIOD FROM JULY 2014 TO JULY 2016 \\ Raúl Arturo Córdova-Verástegui ${ }^{1, a}$, \\ Magdiel José Manuel Gonzales-Menéndez ${ }^{1,2, a, b, c}$, Lucy E. Correa-López ${ }^{1,2}$
}

\begin{abstract}
RESUMEN
Objetivos: Determinar los factores de riesgo maternos relacionados a la presentación de macrosomía fetal en el hospital naval durante julio 2014 - julio 2016. Métodos: Investigación observacional, analítico, caso-control. Siendo los casos recién nacidos macrosómicos (81) (mayor o igual de 4 kg) en el periodo julio 2014 - julio 2016, los controles(81) (peso mayor o igual de $2,5 \mathrm{~kg}$ y menor de $4 \mathrm{~kg}$. El análisis estadístico se realizó con el software SPSS v.22 y Microsoft office Excel. Resultados: De 162 pacientes, 81 presentaron macrosomía fetal. La edad materna varía entre 17 y 42 años, y tiene una media de 29,9 años su asociación estadística indica $O R=4,73$ (IC95\%: 1,509-14,08) p=0.04,la edad gestacional >40 semanas presentó una asociación de OR =0,28 (IC95\%: $0,11-0,72) p=0.006$, los controles prenatales mayores a 6 semanas presento $O R=1,83(I C 95 \%: 0,68-4,93) p=0.22$, la multiparidad obtuvo un $\mathrm{OR}=1,18$ (IC95\%:0,61-2,28) con un valor $\mathrm{p}=0.61$, la talla materna $>1,6$ metro presento $\mathrm{OR}=3,03$ (IC95\%: 1,41-6,53) p=0.004, el antecedente de macrosomía fetal anterior presento OR = 23,23 (IC95\%: $5,32-101,46) \mathrm{p}=0.000$, IMC pregestacional $\mathrm{OR}=2,66(\mathrm{IC} 95 \%: 1,40-5,06) \mathrm{p}=0.003$, la diabetes materna $\mathrm{OR}=2,5$ (IC95\%: 0,48-13,80) p=0.24 y en relación al sexo del bebe OR=2,02 (IC95\%: 1,07-3,78) p=0,027. Conclusión: La edad materna, el antecedente de macrosomía anterior, la talla materna mayor de 1,6 metro, el IMC alto (>26 kg/ $\mathrm{m}^{2}$ ), y el género masculino actúan como factores de riesgo para la presentación de macrosomía fetal.
\end{abstract}

Palabras clave: Factores de riesgo maternos; Recién nacidos macrosómicos. (fuente: DeCS BIREME)

\begin{abstract}
Objective: To determine maternal risk factors related to the presentation of fetal macrosomía at the naval hospital during July 2014 - July 2016. Methods: Observational, analytical, case-control research. As the newborn macrosomic cases (81) (greater than or equal to $4 \mathrm{~kg}$ ) in the period July 2014 to July 2016, controls (81) (weight greater than or equal to $2.5 \mathrm{~kg}$ and less than $4 \mathrm{~kg}$. Was performed with the software SPSS v.22 and Microsoft office Excel. Results: Of 162 patients, 81 presented fetal macrosomía. The maternal age varies between 17 and 42 years and has a mean of 29.9 years its statistical association indicates $O R=P=0.04$, gestational age> 40 weeks had an association of $\mathrm{OR}=0.28(95 \% \mathrm{Cl}: 0.11-0.72) \mathrm{p}=0.006$, controls Prenatales greater than 6 weeks present $\mathrm{OR}=1.83(95 \% \mathrm{Cl}: 0.68-4.93) \mathrm{p}=0.22$, multiparity obtained a $\mathrm{OR}=1.18$ (95\% Cl: $0.61-2.28)$ with a $P=0.61$, the maternal size $>1.6$ meters presented $O R=3.03(95 \% \mathrm{Cl}: 1.41-6.53) \mathrm{p}=0.004$, previous fetal macrosomía present $\mathrm{OR}=23.23$ (95\%: Maternal diabetes $\mathrm{OR}=2.5(95 \% \mathrm{Cl}: 0,95 \% \mathrm{Cl}: 0.40-5.06), \mathrm{p}=0.000, \mathrm{p}$ $=0.000$, pregestational $\mathrm{BMI}=48-13,80) \mathrm{p}=0.24$ and in relation to the sex of the baby $\mathrm{OR}=2.02(95 \% \mathrm{Cl}: 1.07-$ 3.78) $p=0.027$. Conclusions: Maternal age, previous macrosomía, Maternal height greater than 1.6 meters, high BMI (> $\left.26 \mathrm{~kg} / \mathrm{m}^{2}\right)$, and male gender act as risk factors for the presentation of fetal macrosomía.
\end{abstract}

Key words: Maternal risk factors; Macrosomic newborns. (source: MeSH NLM)

\footnotetext{
'Internado Médico del Centro Médico Naval Cirujano Mayor Santiago Távara, Lima-Perú.

${ }^{2}$ Instituto de Investigación en Ciencias Biomédicas, Facultad de Medicina Humana, Universidad Ricardo Palma, Lima, Perú.

a Médico cirujano, b Maestro en Medicina con Mención en Cirugía General, c Especialista en Cirugía General,

¿Economista, Master of Business Administration.
}

Correspondencia: Raúl Arturo Córdova Verástegui. Dirección: Doña Delia 103 Los Rosales, Surco; Lima, Perú. Teléfono: 2712681. Celular: 941683446. Correo: raul.cordova.verastegui@gmail.com

Citar como: Raúl Arturo Córdova-Verástegui, Magdiel José Manuel Gonzales-Menéndez, Lucy E. Correa-López. Factores de riesgo maternos asociados a la presentación de recién nacidos macrosómicos en el Centro Médico Naval Cirujano Mayor Santiago Távara en el período julio 2014 a julio 2016. [Artículo Original]. Rev. Fac. Med. Hum. 2017;17(1):48-55. DOl 10.25176/RFMH.v17.n1.748 


\section{INTRODUCCIÓN}

El esfuerzo del presente trabajo de investigación se dará como parte de la culminación del proceso de formación de estudios de pregrado en el cual se aplicaron los conocimientos adquiridos durante el periodo de formación. Partiendo del conocimiento del impacto de la mortalidad y morbilidad por la presencia de la macrosomía fetal en estudios de países con mayor población y en países con características similares a la nuestra. Nos preguntamos: Cuáles son los factores de riesgo maternos asociados a la presentación de recién nacidos macrosómicos.

\section{OBJETIVOS}

Determinarsilascaracterísticas sociodemográficas, entre ellas la edad materna, IMC pre gestacional, talla materna, antecedente de macrosomía previa, edad gestacional y la paridad; son factores de riesgo para desarrollar macrosomía fetal.

- Determinar si las comorbilidades, entre ellas diabetes mellitus, es un factor de riesgo para desarrollar macrosomía fetal.

- Determinar si el número de controles prenatal $y$ el sexo del bebe son factores de riesgo para presentar macrosomía fetal.

\section{MÉTODOS}

ELtipodeestudio del presentetrabajo es observacional, analítico, de tipo caso y control.

\section{TIPO DE MUESTREO}

Para la estimación de la muestra se aplicó la técnica de la diferencia de proporciones para estudios de casos y controles teniendo un nivel de confianza del $95 \%$ con poder estadístico del $80 \%$. El tamaño muestral estuvo conformado por 81 casos (puérperas con diagnóstico de recién nacidos macrosómicos) comparado con 81 controles (puérperas que tuvieron recién nacidos con peso adecuado para la edad gestacional).

\section{RESULTADOS}

- Con respecto al primer objetivo específico: Determinarsilascaracterísticas sociodemográficas, entre ellas la edad materna, IMC pre gestacional, talla materna, antecedente de macrosomía previa, edad gestacional y la paridad; son factores de riesgo para desarrollar macrosomía fetal.
Tabla 1. Edad materna asociado al desarrollo de Macrosomía fetal en gestantes atendidas en el CEMENA durante julio del 2014 - julio2016.

\begin{tabular}{ccccc}
\hline & \multicolumn{4}{c}{ Macrosomía } \\
& & Si & No & Total \\
\hline Edad & $>35$ años & 16 & 4 & 20 \\
Materna & $\leq 35$ años & $19,8 \%$ & $4,9 \%$ & $12,3 \%$ \\
& & 65 & 77 & 142 \\
\hline Total & & $80,2 \%$ & $95,1 \%$ & $87,7 \%$ \\
\hline OR:4,73 & $X^{2}: 8,23$ p: 0,04 IC (95\%): $1,509-14,08$ \\
\hline
\end{tabular}

Fuente: INICIB-FAMURP/ CEMENA

En la tabla 1 se puede observar que en el grupo de pacientes que presentaron macrosomía fetal como producto de la gestación, el 19,8\% ( $n=16)$ eran mayores de 35 años y el 80,2\% ( $n=65)$ tenían menos de 35 años. Además, en el análisis de su asociación se encuentra un valor obtenido de OR = 4,73 (IC95\%: 1,509-14,08) con un valor $\mathrm{p}=0.04$, lo que nos indica que la edad materna mayor de 35 años actúa como un factor de riesgo para la presentación de macrosomía fetal debido a que el OR es mayor que la unidad y este es significativo debido a que el IC no toma la unidad y el $p$ es $<0,05$.

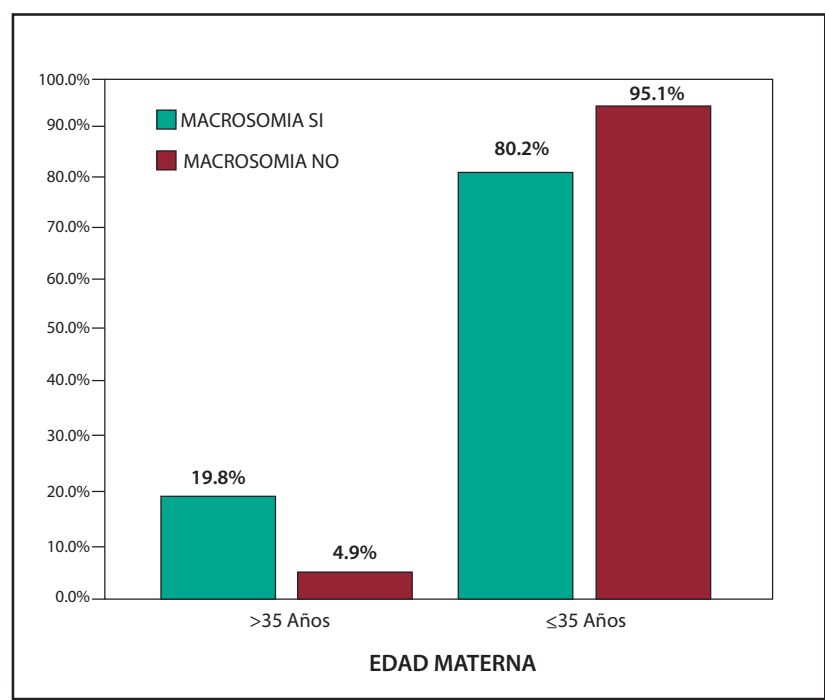

Fuente: INICIB-FAMURP/ CEMENA

Gráfico 1. Edad materna asociado al desarrollo de Macrosomía fetal en gestantes atendidas en el CEMENA durante julio del 2014 - julio2016. 
Tabla 2. Edad gestacional asociado al desarrollo de Macrosomía fetal en gestantes atendidas en el CEMENA durante julio del 2014 - julio2016.

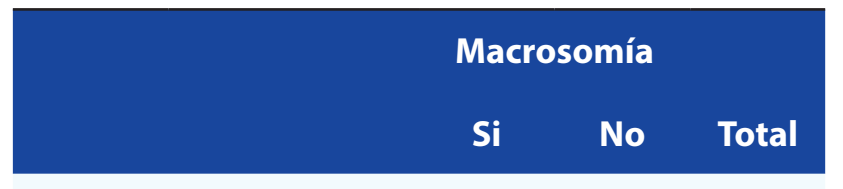

$\begin{array}{ccccc} & >40 \text { Semanas } & 7 & 20 & 27 \\ \text { EdadG } & & & & \\ & \leq 40 \text { Semanas } & 8,6 \% & 24,7 \% & 16,7 \%\end{array}$

\begin{tabular}{|c|c|c|c|c|}
\hline & & 74 & 61 & 135 \\
\hline & & $91,4 \%$ & $75,3 \%$ & $83,3 \%$ \\
\hline Total & $\begin{array}{l}\text { Recuento \% } \\
\text { dentro de } \\
\text { macrosomía }\end{array}$ & $100,0 \%$ & $100,0 \%$ & 162 \\
\hline OF & $X^{2}: 7,5$ P: 0 & 6 IC (9 & $\%): 0,110$ & 0,72 \\
\hline
\end{tabular}

Fuente: INICIB-FAMURP/ CEMENA

En la tabla 2 se puede observar que en el grupo de pacientes que presentaron macrosomía fetal como producto de la gestación, el $8,6 \%(n=7)$ presentaron $>40$ semanas de gestación y el $91,4 \%(n=74)$ tenían menos de 40 semanas de gestación. En el análisis de su asociación se encuentra un valor obtenido de OR =0,28 (IC95\%: $0,11-0,72$ ) con un valor $p=0.006$, lo que nos indica que actúa como un factor protector para la presentación de macrosomía fetal debido a que el OR es menor que la unidad y este es significativo debido a que el IC no toma la unidad y el $p$ es $<0,05$.

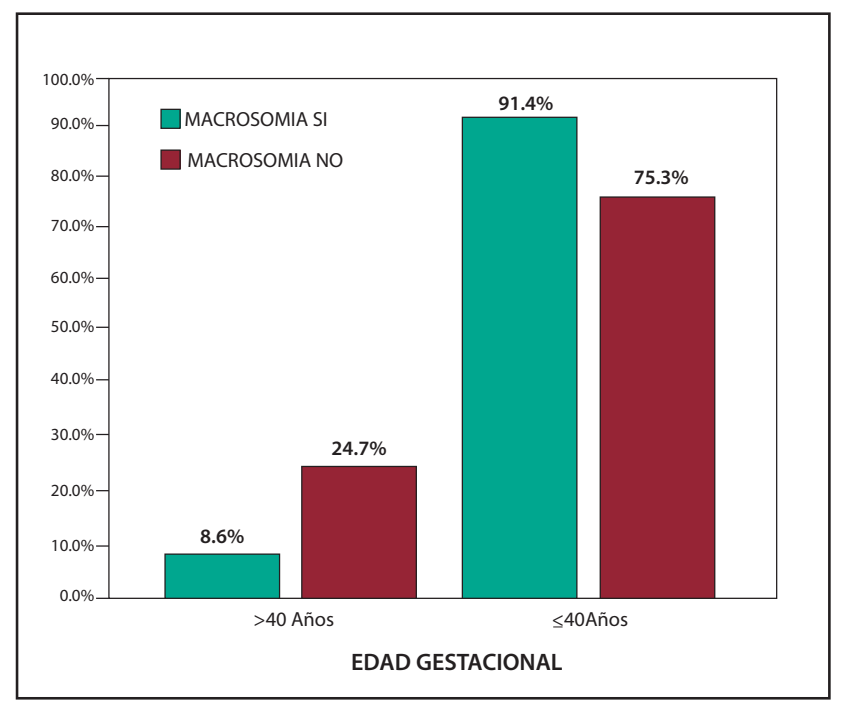

Fuente: INICIB-FAMURP/ CEMENA

Gráfico 2. Edad gestacional asociado al desarrollo de Macrosomía fetal en gestantes atendidas en el CEMENA durante julio del 2014 - julio2016.
Tabla 3. Control pre natal asociado al desarrollo de Macrosomía fetal en gestantes atendidas en el CEMENA durante julio del 2014 - julio2016.

\begin{tabular}{ccccc} 
& \multicolumn{4}{c}{ Macrosomía } \\
& & Si & No & Total \\
\hline CPN & $>6$ CPN & 74 & 69 & 143 \\
& $\leq 6$ CPN & $91,4 \%$ & $85,2 \%$ & $88,3 \%$ \\
& & 7 & 12 & 19 \\
\hline Total & $\begin{array}{c}\text { Recuento } \% \\
\text { dentro de } \\
\text { MACROSOMÍA }\end{array}$ & $8100,0 \%$ & $100,0 \%$ & $100,0 \%$ \\
\hline OR:1,83 & $X^{2}: 1,49$ p: 0,22 IC (95\%):0,68-4,93 \\
\hline
\end{tabular}

Fuente: INICIB-FAMURP/ CEMENA

En la tabla 3 se puede observar que en el grupo de pacientes que presentaron macrosomía fetal como producto de la gestación, el 91,4\% $(n=74)$ presentaron más de 6 controles y el $8,6 \%(n=7)$ tenían menos de 6 controles pre natales. Además, en el análisis de su asociación se encuentra un valor obtenido de $\mathrm{OR}=$ 1,83 (IC95\%: 0,68-4,93) con un valor $p=0.22$, lo que nos indica que el número de controles prenatales mayores de 6 oportunidades no actúa como un factor riesgo para la presentación de macrosomía fetal debido a que estadísticamente no es significativo debido a que el IC toma la unidad y el $p$ es $>0,05$.

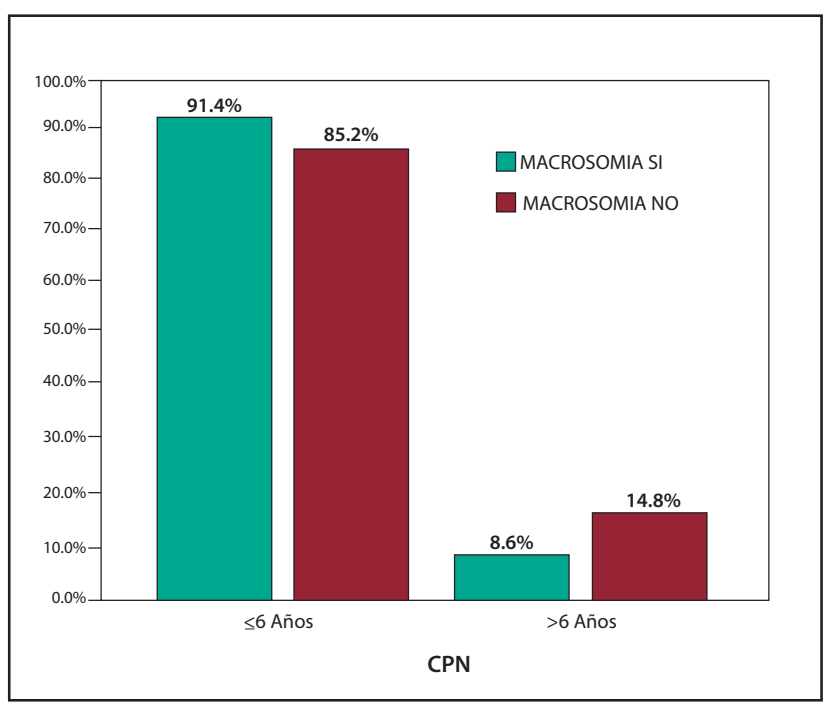

Fuente: INICIB-FAMURP/ CEMENA

Gráfico 3. Control pre natal asociado al desarrollo de Macrosomía fetal en gestantes atendidas en el CEMENA durante julio del 2014 - julio2016. 
Tabla 4. La paridad asociada al desarrollo de Macrosomía fetal en gestantes atendidas en el CEMENA durante julio del 2014 - julio2016.

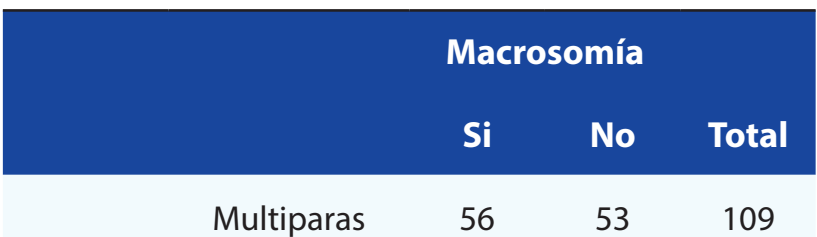

Paridad

Primiparas $\quad 69,1 \% \quad 65,4 \% \quad 67,3 \%$

$\begin{array}{ccccc} & & 25 & 28 & 53 \\ & & 30,9 \% & 34,6 \% & 32,7 \% \\ \text { Total } & \begin{array}{c}\text { Recuento } \% \\ \text { dentro de }\end{array} & 81 & 81 & 162 \\ & \text { MACROSOMÍA } & 100,0 \% & 100,0 \% & 100,0 \%\end{array}$

OR:1,18 $x^{2}: 0,25$ p: 0,61 IC (95\%):0,61-2,28

Fuente: INICIB-FAMURP/ CEMENA

En la tabla 4 se puede observar que en el grupo de pacientes que presentaron macrosomía fetal como producto de la gestación, el $69,1 \%(n=56)$ tuvieron como antecedente ser multíparas y el 30,9\% $(n=25)$ eran primíparas. Además, en el análisis de su asociación se encuentra un valor obtenido de $\mathrm{OR}=1,18$ (IC95\%: $0,61-2,28$ ) con un valor $p=0.61$, lo que nos indica que multiparidad no actúa como un factor riesgo para la presentación de macrosomía fetal debido a que estadísticamente no es significativo debido a que el IC toma la unidad y el $p$ es $>0,05$.

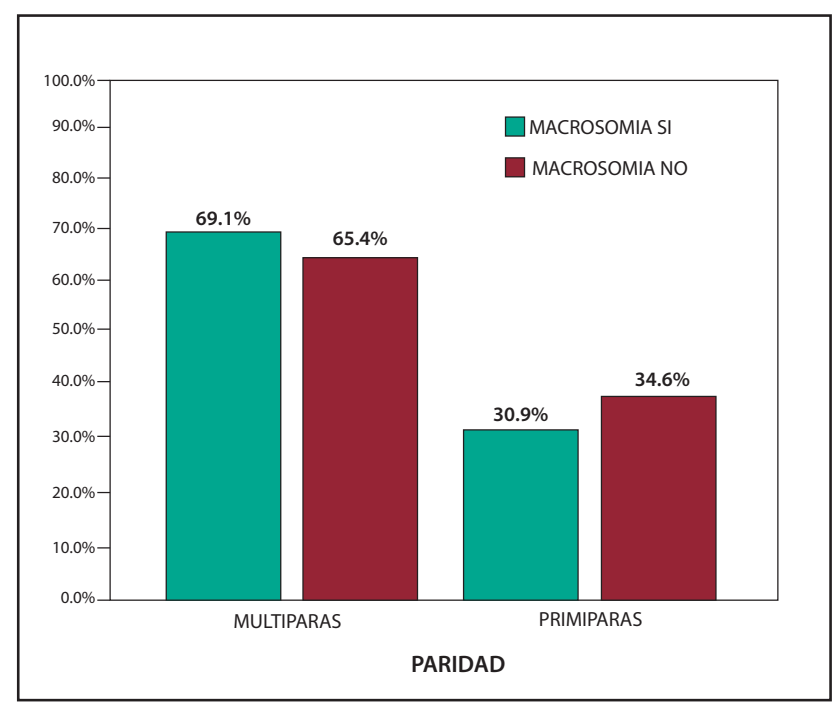

Fuente: INICIB-FAMURP/ CEMENA

Gráfico 4. Paridad asociada al desarrollo de Macrosomía fetal en gestantes atendidas en el CEMENA durante julio del 2014 julio2016.
Tabla 5. Talla materna asociado al desarrollo de Macrosomía fetal en gestantes atendidas en el CEMENA durante julio del 2014 - julio2016.

\begin{tabular}{|c|c|c|c|c|}
\hline & & \multicolumn{2}{|c|}{ Macrosomía } & \multirow[b]{2}{*}{ Total } \\
\hline & & Si & No & \\
\hline \multirow{4}{*}{ Talla M } & $\geq 1,6$ Metros & 28 & 12 & 40 \\
\hline & $<1,6$ Metros & $34,6 \%$ & $14,8 \%$ & $24,7 \%$ \\
\hline & & 53 & 69 & 122 \\
\hline & & $65,4 \%$ & $85,2 \%$ & $75,3 \%$ \\
\hline \multirow[t]{2}{*}{ Total } & $\begin{array}{c}\text { Recuento \% } \\
\text { dentro de }\end{array}$ & 81 & 81 & 162 \\
\hline & MACROSOMÍA & $100,0 \%$ & $100,0 \%$ & $100,0 \%$ \\
\hline OR: & \multicolumn{4}{|c|}{$3 x^{2}: 8,49$ p: 0,004 IC (95\%):1,41-6,52 } \\
\hline
\end{tabular}

Fuente: INICIB-FAMURP/ CEMENA

En la tabla 5 se puede observar que en el grupo de pacientes que presentaron macrosomía fetal como producto de la gestación, el $34,6 \%(n=28)$ presentaban una talla mayor de 1,6 metros y el $65,4 \%(n=53)$ tenían menos de 1,6 metros. Además, en el análisis de su asociación se encuentra un valor obtenido de $\mathrm{OR}=3,03$ (IC95\%: 1,41-6,53) con un valor $\mathrm{p}=0.004$, lo que nos indica que la talla materna mayor de 1,6 metros actúa como un factor de riesgo para la presentación de macrosomía fetal debido a que presenta un OR mayor que la unidad y este es estadísticamente significativo debido a que el IC no toma la unidad y el $p$ es $<0,05$.

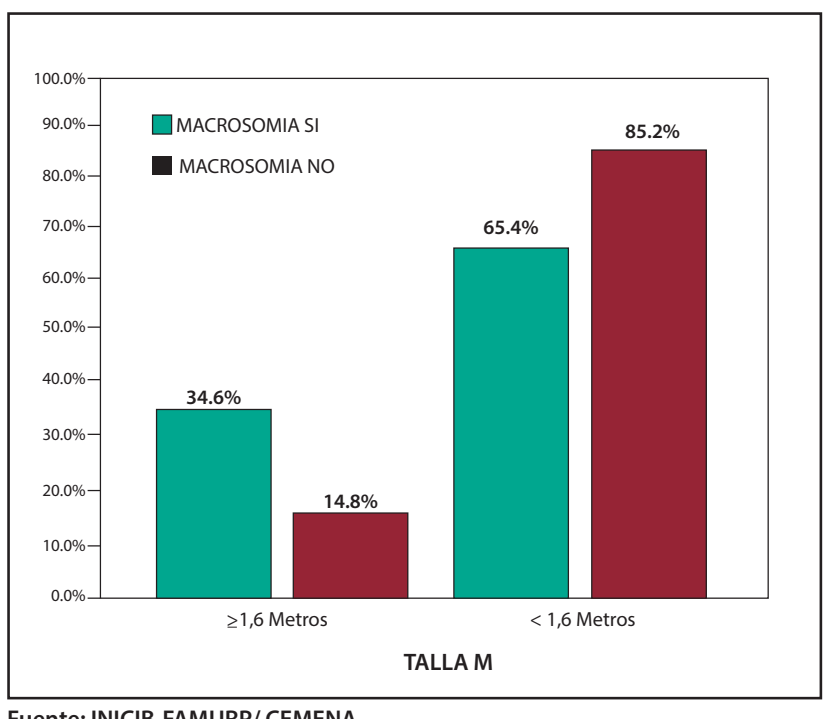

Fuente: INICIB-FAMURP/ CEMENA

Gráfico 5. Talla materna asociada al desarrollo de Macrosomía fetal en gestantes atendidas en el CEMENA durante julio del 2014 - julio2016. 
Tabla 6. Antecedente de macrosomía anterior asociado al desarrollo de Macrosomía fetal en gestantes atendidas en el CEMENA durante julio del 2014 - julio2016.

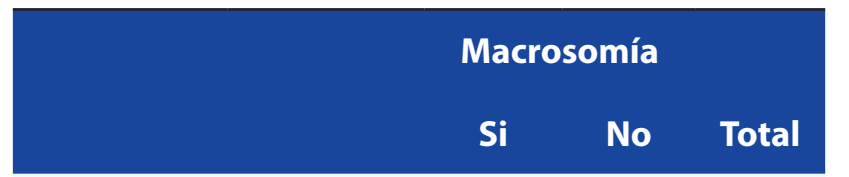

$\begin{array}{lcccc}\begin{array}{l}\text { ANTECEDENTE } \\ \text { DE }\end{array} & \text { SI } & 30 & 2 & 32 \\ \begin{array}{l}\text { MACROSOMÍA } \\ \text { ANTERIOR }\end{array} & \text { NO } & 37,0 \% & 2,5 \% & 19,8 \%\end{array}$

\begin{tabular}{ccccc} 
& & 51 & 79 & 130 \\
\hline Total & $63,0 \%$ & $97,5 \%$ & $80,2 \%$ \\
& $\begin{array}{c}\text { Recuento } \% \\
\text { dentro de } \\
\text { MACROSOMÍA }\end{array}$ & 81 & 81 & 162 \\
\hline & $100,0 \%$ & $100,0 \%$ & $100,0 \%$
\end{tabular}

\section{OR:23,23 $x^{2}: 30,5$ p: 0,000 IC (95\%): 5,32-101,46}

Fuente: INICIB-FAMURP/ CEMENA

En la tabla 6 se puede observar que en el grupo de pacientes que presentaron macrosomía fetal como producto de la gestación, el $37 \%(n=30)$ tienen como antecedente haber presentado macrosomía fetal anterior y el $63 \%(n=51)$ no presentaban antecedente de macrosomía fetal anterior. Además, en el análisis de su asociación se encuentra un valor obtenido de $\mathrm{OR}=$ 23,23 (IC95\%: 5,32-101,46) con un valor $p=0.000$, lo que nos indica que el antecedente de haber presentado macrosomía fetal anterior actúa como un factor de riesgo para la presentación de macrosomía fetal debido a que presenta un OR mayor que la unidad y este es estadísticamente significativo debido a que el IC no toma la unidad y el $p$ es $<0,05$.

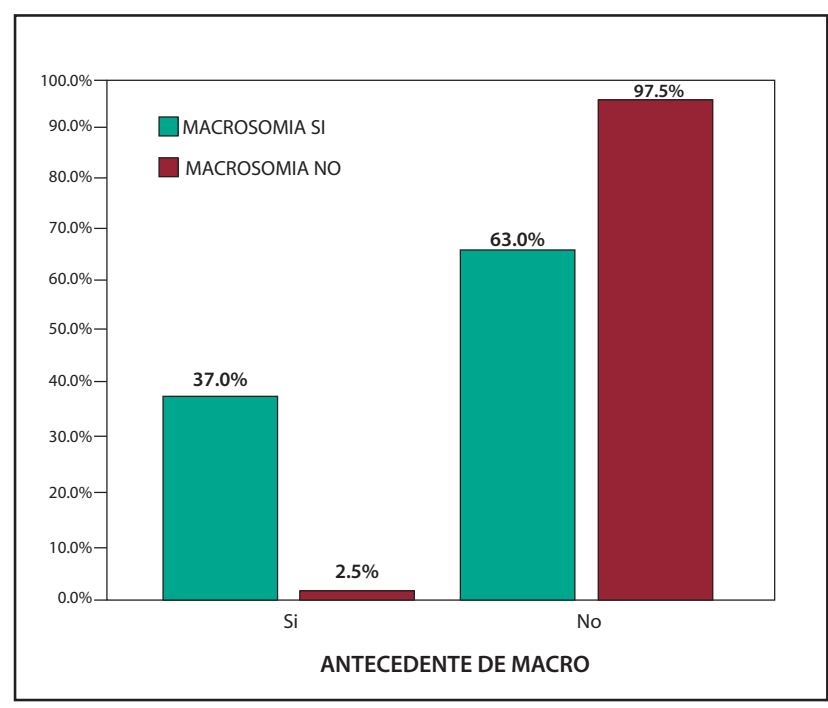

Fuente: INICIB-FAMURP/ CEMENA

Gráfico 6. Antecedente de macrosomía fetal anterior asociado al desarrollo de Macrosomía fetal en gestantes atendidas en el CEMENA durante julio del 2014 - julio2016.
Tabla 7. IMC pregestacional asociado al desarrollo de Macrosomía fetal en gestantes atendidas en el CEMENA durante julio del 2014 - julio2016.

\begin{tabular}{|c|c|c|c|c|}
\hline & & \multicolumn{2}{|c|}{ Macrosomía } & \multirow[b]{2}{*}{ Total } \\
\hline & & Si & No & \\
\hline \multirow[t]{4}{*}{$\begin{array}{l}\text { IMC } \\
\text { PREGESTACIONAL }\end{array}$} & $\begin{array}{l}\text { Alto IMC: } \\
>26 \mathrm{Kg} / \mathrm{m} 2\end{array}$ & 44 & 25 & 69 \\
\hline & $\begin{array}{l}\text { Bajo y } \\
\text { normal IMC: } \\
<26 \mathrm{Kg} / \mathrm{m} 2\end{array}$ & $54,3 \%$ & $30,9 \%$ & $42,6 \%$ \\
\hline & & 37 & 56 & 93 \\
\hline & & $45,7 \%$ & $69,1 \%$ & $57,4 \%$ \\
\hline \multirow[t]{2}{*}{ Total } & $\begin{array}{l}\text { Recuento \% } \\
\text { dentro de }\end{array}$ & 81 & 81 & 162 \\
\hline & MACROSOMÍA & $100,0 \%$ & $100,0 \%$ & $100,0 \%$ \\
\hline
\end{tabular}

OR: $2,66 \quad x^{2}: 9,11$ p: 0,003 IC (95\%): 1,40-5,06

Fuente: INICIB-FAMURP/ CEMENA

En la tabla 7 se puede observar que en el grupo de pacientes que presentaron macrosomía fetal como producto de la gestación, el 54,3\% $(n=44)$ presentaron un IMC pre gestacional alto $(>26 \mathrm{Kg} / \mathrm{m} 2)$ y el $45,7 \%$ $(\mathrm{n}=37)$ presentaron un IMC bajo y normal $(:<26 \mathrm{Kg} / \mathrm{m} 2)$. Además, en el análisis de su asociación se encuentra un valor obtenido de OR = 2,66 (IC95\%: 1,40-5,06) con un valor $p=0.003$, lo que nos indica que el presentar IMC alto (>26 Kg/m2) actúa como un factor de riesgo para la presentación de macrosomía fetal debido a que presenta un OR mayor que la unidad y este es estadísticamente significativo debido a que el IC no toma la unidad y el $p$ es $<0,05$.

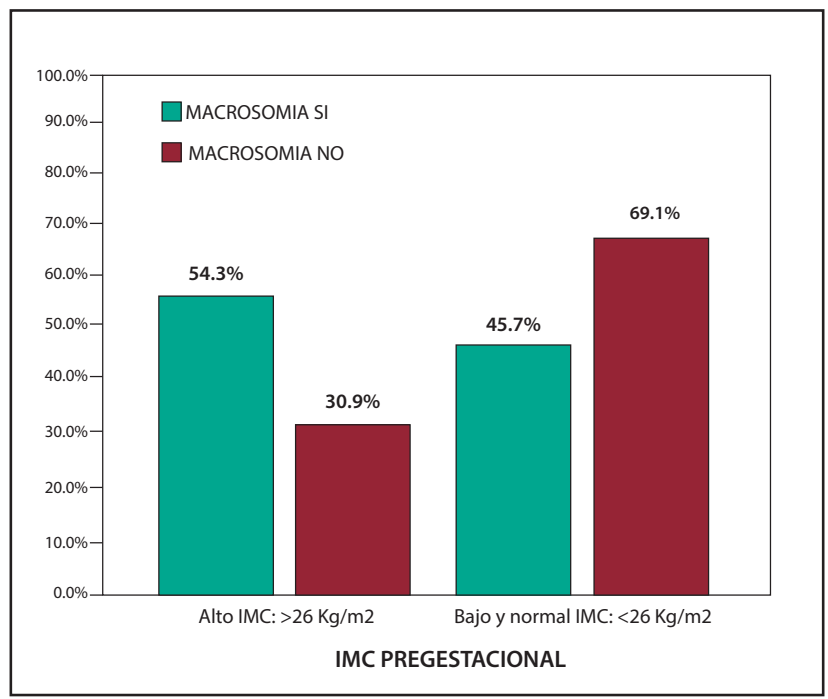

Fuente: INICIB-FAMURP/ CEMENA

Gráfico 7. IMC pregestacional asociado al desarrollo de Macrosomía fetal en gestantes atendidas en el CEMENA durante julio del 2014 - julio2016. 
Tabla 8. Diabetes materna asociado al desarrollo de Macrosomía fetal en gestantes atendidas en el CEMENA durante julio del 2014 - julio2016.

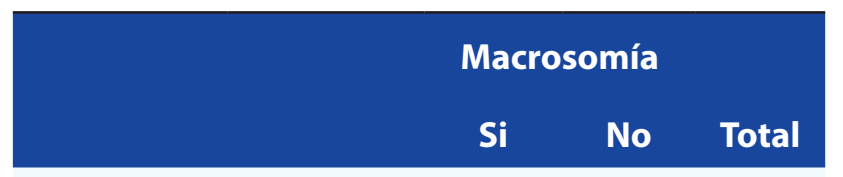

$\begin{array}{lcccc} & \text { Si } & 5 & 2 & 7 \\ \text { Diabetes } & \text { No } & 6,2 \% & 2,5 \% & 4,3 \% \\ & & 76 & 79 & 155 \\ \text { Total } & 93,8 \% & 97,5 \% & 95,7 \% \\ & \begin{array}{c}\text { Recuento } \% \\ \text { dentro de } \\ \text { MACROSOMÍA }\end{array} & 100,0 \% & 100,0 \% & 100,0 \%\end{array}$

OR: $2,5 \quad x^{2}: 1,34$ p: 0,24 IC (95\%): 0,48-13,80

Fuente: INICIB-FAMURP/ CEMENA

En la tabla 8 se puede observar que en el grupo de pacientes que presentaron macrosomía fetal como producto de la gestación, el $6,2 \%(n=5)$ presentaron diabetes materna y el $93,8 \%(n=76)$ no presentaron diabetes materna. Además, en el análisis de su asociación se encuentra un valor obtenido de $\mathrm{OR}=$ 2,5 (IC95\%: 0,48-13,80) con un valor $p=0.24$, lo que nos indica que el presentar por parte de la madre diabetes no actúa como un factor de riesgo para la presentación de macrosomía fetal debido a que no es estadísticamente significativo debido a que el IC toma la unidad y el $p$ es $>0,05$.

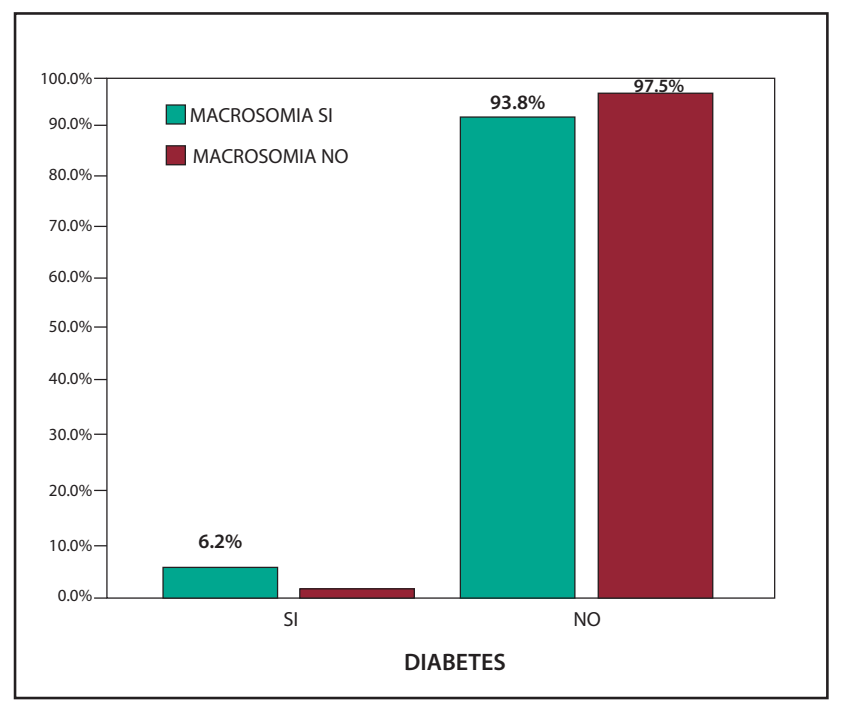

Fuente: INICIB-FAMURP/ CEMENA

Gráfico 8. Diabetes materna asociado al desarrollo de Macrosomía fetal en gestantes atendidas en el CEMENA durante julio del 2014 - julio2016.
Tabla 9. Sexo del bebe asociado al desarrollo de Macrosomía fetal en gestantes atendidas en el CEMENA durante julio del 2014 - julio2016.

\begin{tabular}{ccccc} 
& \multicolumn{4}{c}{ Macrosomía } \\
& Si & No & Total \\
\hline $\begin{array}{c}\text { Sexo del } \\
\text { Bebe }\end{array}$ & Masculino & 51 & 37 & 88 \\
& Femenino & $63,0 \%$ & $45,7 \%$ & $54,3 \%$ \\
\hline Total & 30 & 44 & 74 \\
\hline OR:2,02 & $\begin{array}{c}\text { Recuento } \% \\
\text { dentro de }\end{array}$ & 81 & 81 & 162 \\
& $\begin{array}{c}\text { MACROSOMIA } \\
\text { X }: 4,87 \text { p: } 0,027 \text { IC (95\%): } 1,07-3,78\end{array}$ \\
\hline
\end{tabular}

Fuente: INICIB-FAMURP/ CEMENA

En la tabla 9 se puede observar que en el grupo de pacientes que presentaron macrosomía fetal como producto de la gestación, el 63,0\% $(n=51)$ tuvieron un recién nacido masculino y el $37,0 \%(n=30)$ tuvieron un recién nacido femenino. Además, en el análisis de asociación se encuentra un valor obtenido de $\mathrm{OR}=$ 2,02 (IC95\%: 1,07-3,78) con un valor $p=0,027$, lo que nos indica que el presentar como producto un bebe del género masculino actúa como un factor de riesgo para la presentación de macrosomía fetal debido a que presenta un OR por encima de la unidad y es estadísticamente significativo debido a que el IC no toma la unidad y el $p$ es $<0,05$.

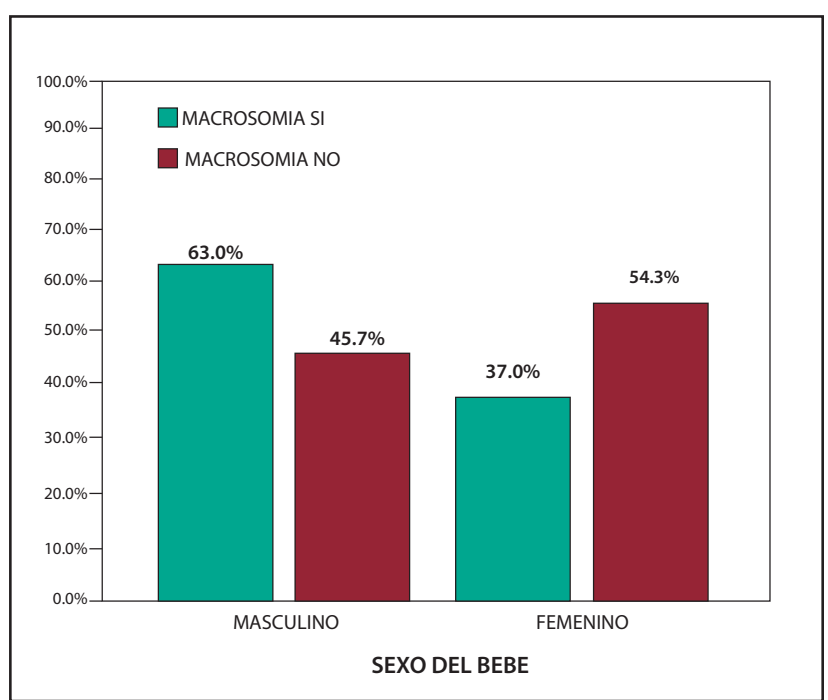

Fuente: INICIB-FAMURP/ CEMENA

Gráfico 9. Sexo del bebe asociado al desarrollo de Macrosomía fetal en gestantes atendidas en el CEMENA durante julio del 2014 - julio2016. 
Tabla 10. Tabla general de estimación de riesgo, intervalo de confianza y significancia (p).

\begin{tabular}{|c|c|c|c|c|c|c|}
\hline \multirow{2}{*}{ Variable } & \multirow{2}{*}{ Indicador } & \multicolumn{2}{|c|}{ Macrosomía } & \multirow{2}{*}{ OR } & \multirow{2}{*}{ Validez(P) } & \multirow{2}{*}{ IC (95\%) } \\
\hline & & Si & No & & & \\
\hline \multirow{3}{*}{ Edad Materna } & >35 años & $19,8 \%$ & $4,9 \%$ & \multirow{3}{*}{4.73} & \multirow{3}{*}{0,04} & \multirow{3}{*}{$1.50-14,08$} \\
\hline & & & & & & \\
\hline & $\leq 35$ años & $80,2 \%$ & $91,5 \%$ & & & \\
\hline \multirow{2}{*}{$\begin{array}{l}\text { Edad } \\
\text { gestacional }\end{array}$} & $>40$ Semanas & $8,6 \%$ & $24,7 \%$ & \multirow{2}{*}{0.289} & \multirow{2}{*}{0.006} & \multirow{2}{*}{$0.11-0,72$} \\
\hline & $\leq 40$ Semanas & $91,4 \%$ & $75,3 \%$ & & & \\
\hline \multirow{3}{*}{ IMC } & Alto índice & $54,3 \%$ & $30,9 \%$ & \multirow{3}{*}{2.66} & \multirow{3}{*}{0.003} & \multirow{3}{*}{$1.40-5.06$} \\
\hline & Baio índice o & & & & & \\
\hline & normal & $45,7 \%$ & $69,1 \%$ & & & \\
\hline \multirow{3}{*}{ Talla materna } & $\geq 1,6$ Metros & 34,6 & $14,8 \%$ & \multirow{3}{*}{3.03} & \multirow{3}{*}{0.004} & \multirow{3}{*}{$1.41-6.52$} \\
\hline & & & & & & \\
\hline & $<1,6$ Metros & $65,4 \%$ & $85,2 \%$ & & & \\
\hline \multirow{3}{*}{ Paridad } & Multíparas & $69,1 \%$ & $65,4 \%$ & \multirow{3}{*}{1.18} & \multirow{3}{*}{0.61} & \multirow{3}{*}{$0.61-2.28$} \\
\hline & & & & & & \\
\hline & Primíparas & $30,9 \%$ & $34,6 \%$ & & & \\
\hline \multirow{2}{*}{$\begin{array}{l}\text { Antecedentes } \\
\text { Macrosómicos }\end{array}$} & $\mathrm{Si}$ & $37,0 \%$ & $2,5 \%$ & \multirow{2}{*}{23.23} & \multirow{2}{*}{0.000} & \multirow{2}{*}{$\begin{array}{c}5.32- \\
101.46\end{array}$} \\
\hline & No & $63,0 \%$ & $97,5 \%$ & & & \\
\hline \multirow{2}{*}{$\begin{array}{l}\text { Controles } \\
\text { prenatales }\end{array}$} & $\geq 6 \mathrm{CPN}$ & $91,4 \%$ & $85,2 \%$ & \multirow{2}{*}{1.83} & \multirow{2}{*}{0.22} & \\
\hline & $<6 \mathrm{CPN}$ & $8,6 \%$ & $14,8 \%$ & & & \\
\hline Diabetes & $\mathrm{Si}$ & $6,2 \%$ & $2,5 \%$ & & & \\
\hline Materna & No & $93.8 \%$ & $97.5 \%$ & 2.5 & 0.24 & $0.48-13.80$ \\
\hline & Masculino & $63,0 \%$ & $65,7 \%$ & & & \\
\hline Sexo del bebe & & & & 2.02 & 0.027 & $1.07-3.78$ \\
\hline & Femenino & $37,0 \%$ & $34,3 \%$ & & & \\
\hline
\end{tabular}

Fuente: INICIB-FAMURP/ CEMENA

\section{DISCUSIÓN}

Se evidencia en nuestros resultados que la edad materna mayor de 35 años es un factor de riesgo en relación a la presentación de macrosomía fetal, debido a que se obtuvo un intervalo de confianza que no toma la unidad y el valor de $p$ es $<0.05$ el cual también se evidencia en trabajos realizados como en de Shouyong Gu y col. Donde determinan que la edad materna es un factor de riesgo ya que presenta OR 1.04 y IC95\% de 1.02-1.05.

La edad gestacional actúa como un factor protector para la presentación de macrosomía fetal debido a que el OR es menor que la unidad y este es significativo debido a que el IC no toma la unidad y el $p$ es $<0,05$, el cual muestra datos totalmente contrarios a trabajos realizados como el de Arpasi Tipula y col. donde la edad gestacional se presenta frente a la macrosomía fetal con un OR: 7,42 y un IC 95\% de 3,3-16,2.

Los controles prenatales no actúan como factor de riesgo para presentar macrosomía fetal, puesto que esta asociación no fue estadísticamente significativa Estos resultados se asemejan al estudio de Chávez y col. que encontraron el $69,80 \%$ que representa un control prenatal adecuado en el grupo de los casos, guardando relación con la macrosomía.

La paridad no actúa como factor de riesgo, Estos 
resultados no guarda relación con los encontrados en estudios que sí presentan relación con la macrosomía como es el caso del estudio de Ávila y col. en donde el número de gestaciones de las madres de los casos fue en promedio de 2,86 y en los controles de 2,32 con un grado de asociación de $p=0,01$, siendo significativo para la relación entre la multiparidad y los recién nacidos macrosómicos.

La talla materna actúa como un factor de riesgo para presentar macrosomía fetal, se pueden encontrar estudios en los cuales se hallarán valores mayores que demuestran asociación entre las variables como el de Farfán. en él se puede evidenciar una asociación estadísticamente significativa entre la talla materna y macrosomía en el recién nacido, con un valor de $\mathrm{OR}=$ 2,05; al igual que en el estudio de Arpasi, en el cual el $73,30 \%$ de las madres tienen talla alta, asociándose ésta a la macrosomía fetal con valor de $\mathrm{p}=0,002$.

La IMC gestacional actúa como un factor de riesgo para presentar macrosomía fetal, Estos resultados coinciden con los de Bove, que obtuvo una media del IMC pregestacional de 24,09 $\pm 4,28$, siendo estadísticamente significativo con un valor de $p<0,001$ al relacionar el peso pregestacional con la macrosomía. También Zonana, encontró relación significativa entre el peso pregestacional y macrosomía en el recién nacido $(\mathrm{RM}=1,8$ IC 95\%) en el que puérperas con sobrepeso previo al embarazo presentaron RM 2,5 (IC 95\% 1,1-5,6).

El antecedente de macrosomía fetal actúa como un factor de riesgo para presentar macrosomía fetal, estos resultados concuerdan con estudios con valores mayores como el de Razeto Factores asociados a morbimortalidad del recién nacido macrosómico en el Hospital Nacional Daniel Alcides Carrión, enerodiciembre del 2010, donde se encuentra una relación significativa $(p=0,0012)$.

El antecedente del genero del bebe actúa como un factor de riesgo para presentar macrosomía fetal, dichos resultados coinciden con los dados por Mahnaz Mardani y col. quienes señalan que La mayoría de los niños macrosómicos eran varones. Los resultados mostraron que la incidencia de macrosomía en los niños varones es 2,331 veces mayor que las mujeres neonatas.

La diabetes materna no actúa como un factor de riesgo para presentar macrosomía fetal por no ser significativa en cambio en el estudio de Paredes Torres se aprecia que, de las madres diabéticas, el $83 \%$ tuvo hijos macrosómicos y el 17\% tuvieron hijos no macrosómicos. Al igual que lo descrito en la literatura médica y en estudios anteriores realizados por Cutié, Pacora, entre otros, podemos observar una relación existente entre la diabetes materna y la macrosomía fetal.

\section{RECOMENDACIONES}

Intensificar el control prenatal en aquellas pacientes mayores a 35 años, IMC alto, con el antecedente de haber presentado macrosomía fetal anterior, con una talla materna mayor de 1,6 metros debido a que resultaron ser factores de riesgo para presentar macrosomía fetal.

Realizar estudios posteriores relacionados al tema con la finalidad de determinar otras variables que se puedan asociar a la macrosomía fetal; para ello se recomienda tener una población más numerosa para que lo resultados sean representativos.

Intensificar el estudio en relación a la multiparidad y la diabetes materna en función a los problemas de macrosomía fetal debido a que muchos trabajos no son concluyentes.

\section{Financiamiento: Autofinanciado.}

Conflicto de interés: Los autores declaran no tener conflictos de interés en la publicación de este artículo.

Recibido: 02 de marzo del 2017

Aprobado: 20 de marzo del 2017

\section{REFERENCIAS BIBLIOGRÁFICAS}

\footnotetext{
1. Jazayeri Allahyar, PhD. Macrosomía. EMedicine 2007. Direccion de internet: www.emedicine.com/med/topic3279.htm

2. Sincleir Be, Hainsworth OT. Los niños macrosómicos no son todos iguales Aust N Z J Obstet Gynaecol 2007; 47: 101-105.

3. Romaña MC, Paralisis obstetrica del plexo braquial, J Gynecol Obstet Bio Reprod (Paris) 2000; 29 (1 Suppl): 33-35.

4. Carlos C, Racault A, de Gamarra E, Wallet A.El recien nacido macrosomico en la maternidad: actitud practica. J Gynecol Obstet Biol Reprod (Paris) 2000: 29 (1 Suppl): 25-32.

5. Lawrence E. Una cuestion de tamaño: parte 2 , evaluacion de los grandes neonates de edad gestacional.. Adv Neonatal Care 2007; 7: 187-97.

6. Hawdon J. De Rooy L, Factores nutricionales que afectan la adaptacion metabolica postnatal de niños pequeños y grandes de edad. Pediatrics 2002; 109: E42.

7. Reganault, Sean W. Timothy R.HLimesand, William W. Hay Jr. Factores que influyen en el crecimiento fetal vol 2. No6. June 2001
}

8. Chiesa C, Osborn JF, Haass C, Natale F, Spinelli M, Scapillati E et al. Ghrelin, Leptin, IGF-1, IGFBP-3 and Concentracion de insulina en el nacimiento. Existe alguna relaacion entre el crecimiento fetal y la antropometria neonatal. Clin Chem. 2008 Jan 17 [Epub ahead of print]

9. Campaigne A., Conway D Deteccion y prevencion de la macrosomía fetal Obstet Gynecol Clin N Am 2007; 34: 309-322.

10. Goffinet FDificultades en el reconocimiento prenatal de macrosomía fetal. J Gynecol Obstet Biol Reprod (Paris) 2000; 29(1 Suppl):13- 19

11. Torres González, Cristóbal; Hernández Barrios, Esteban; Moreno Torres, Joselin; Rodríguez Cabrera, Angel; Vázquez Martínez. Factores de riesgo para macrosomía en recién nacidos hijos de madre con diabetes gestacional MediSur, vol. 4, núm. 1, 2006, pp. 13-18

12.-Ramos MM Batista DF, Nobrega, TG, FATORES DE RISCO PARA MACROSOMÍA EM RECEM -NASCIDOS DE UMA MATERNIDA DE -ESCOLA NO NORDESTE DO BRASIL. Rev Bras Ginecol Obstet 2009; 31(5): 241-8 